\title{
gRNA validation for wheat genome editing with the CRISPR-Cas9 system
}

\author{
Taj Arndell ${ }^{*}$ (D), Niharika Sharma ${ }^{2}$, Peter Langridge ${ }^{3}$, Ute Baumann ${ }^{3}$, Nathan S. Watson-Haigh ${ }^{4}$ and Ryan Whitford ${ }^{3}$
}

\begin{abstract}
Background: The CRISPR-Cas9 system is a powerful and versatile tool for crop genome editing. However, achieving highly efficient and specific editing in polyploid species can be a challenge. The efficiency and specificity of the CRISPRCas9 system depends critically on the gRNA used. Here, we assessed the activities and specificities of seven gRNAs targeting 5-enolpyruvylshikimate-3-phosphate synthase (EPSPS) in hexaploid wheat protoplasts. EPSPS is the biological target of the widely used herbicide glyphosate.

Results: The seven gRNAs differed substantially in their on-target activities, with mean indel frequencies ranging from $0 \%$ to approximately $20 \%$. There was no obvious correlation between experimentally determined and in silico predicted on-target gRNA activity. The presence of a single mismatch within the seed region of the guide sequence greatly reduced but did not abolish gRNA activity, whereas the presence of an additional mismatch, or the absence of a PAM, all but abolished gRNA activity. Large insertions ( $\geq 20 \mathrm{bp}$ ) of DNA vector-derived sequence were detected at frequencies up to $8.5 \%$ of total indels. One of the gRNAs exhibited several properties that make it potentially suitable for the development of non-transgenic glyphosate resistant wheat.

Conclusions: We have established a rapid and reliable method for gRNA validation in hexaploid wheat protoplasts. The method can be used to identify gRNAs that have favourable properties. Our approach is particularly suited to polyploid species, but should be applicable to any plant species amenable to protoplast transformation.
\end{abstract}

Keywords: Genome editing, CRISPR-Cas9, gRNA, Wheat, Triticum aestivum, Protoplasts, EPSPS

\section{Background}

Genome editing technologies enable the targeted and precise modification of plant genomes via the creation and subsequent repair of site-specific DNA double-strand breaks (DSBs) [1]. Over the last few years, the field of genome editing has been revolutionised by the introduction of the CRISPR (clustered regularly-interspaced short palindromic repeats)-Cas9 (CRISPR associated protein) system [2-7]. This system consists of the Cas9 endonuclease in complex with a small guide RNA (gRNA) that is engineered to target a specific site in the genome. The target site is defined by a 20 nucleotide guide sequence at the $5^{\prime}$ end of the gRNA, making programming of the system relatively straightforward. For the system to function, the target site must be located immediately $5^{\prime}$ to a protospacer adjacent motif (PAM) whose canonical form is $5^{\prime}$-NGG-3' (for SpCas9 from Streptococcus pyogenes).

\footnotetext{
* Correspondence: taj.arndell@csiro.au

'Present address: CSIRO, Agriculture and Food, Canberra, ACT, Australia

Full list of author information is available at the end of the article
}

The PAM is on the strand opposite to the strand bound by the gRNA. Site-specific DSBs generated by Cas9 are typically repaired through either of two competing pathways: non-homologous end joining (NHEJ) or homology directed repair (HDR). NHEJ, which is the predominant repair pathway in somatic plant cells [8], is error-prone and often produces small insertions/deletions (indels) that result in gene knockout (e.g. through frame shift or the creation of a premature stop codon) [9-12]. Alternatively, if an exogenous DNA donor template with homologous ends is delivered to the cell, then precise modifications (sequence insertion or replacement) can be made through HDR [9, 13-18]. If the homologous ends of the donor template are short (5-25 bp), then the DSB may be repaired through microhomology-mediated end joining (MMEJ) [19]. Due to its simplicity, flexibility, and high specificity, the CRISPR-Cas9 system has been and continues to be rapidly adopted by the plant research community for basic research and crop improvement. 
Although the CRISPR-Cas9 system has been successfully applied to many model and crop plants, editing efficiencies have varied greatly and in many cases have been lower than one would desire. In particular, gene knockout via NHEJ tends to be relatively inefficient in polyploidy species due to genetic redundancy [20-22]. Furthermore, the inherent low frequency of HDR remains a major challenge in plant genome editing [23]. In addition, despite the high specificity of the CRISPR-Cas9 system, off-target mutations can occur at sites that have sequence similarity to the target site [24-26], especially when there are no mismatches in the PAM-proximal 8-12 nucleotide 'seed region' of the guide sequence [27-30]. Such off-target sites may be present in non-target genes or non-target alleles. Off-target mutations are undesirable as they may confound results and/or produce impaired phenotypes, in which case they must be removed by backcrossing. Consequently, much effort has been directed toward improving the efficiency and specificity of the CRISPR-Cas9 system in plants and other organisms.

It is well-established that the efficiency and specificity of the CRISPR-Cas9 system depends critically on target site selection, as well as certain sequence features of the gRNA. Thus, an effective strategy for achieving efficient and specific editing is to use gRNAs that exhibit high activity and specificity. A number of bioinformatics tools have been developed for the in silico prediction of ontarget and/or off-target gRNA activity [31-36]. Some of these tools can provide reliable predictions for potential off-target sites in a limited number of species, and predictions for on-target gRNA activity can reduce the time spent on gRNA screening [37]. However, the predictions are not always accurate, and the development and independent validation of these tools has been based on data obtained from non-plant species. Therefore, it is prudent to carry out experimental validation of gRNAs prior to commencing plant transformation experiments that require substantial investment of time and resources.

Here, we propose and test a strategy for assessing gRNA activity and specificity, using seven gRNAs targeting 5enolpyruvylshikimate-3-phosphate synthase (EPSPS) in hexaploid wheat (Triticum aestivum). EPSPS is an ideal target for editing via HDR, as several well-characterised amino acid substitutions in this gene are known to confer resistance to the widely used herbicide glyphosate [38]. Furthermore, in wheat there is an opportunity to take advantage of its hexaploid nature by performing homoeoallele-specific editing, thereby potentially avoiding the severe yield penalty associated with homozygous amino acid substitutions and loss-of-function mutations in EPSPS [39-41]. Therefore, one of our aims was to identify a highly active and homoeoallele-specific gRNA targeting ESPSP. Three of our gRNAs were designed to be homoeoallele-specific based on the presence of single nucleotide polymorphisms (SNPs) between the three homoeologous copies of EPSPS. This allowed us to determine the frequency of off-target mutagenesis. The seven gRNAs were rigorously evaluated through transient expression of CRISPR-Cas9 reagents in wheat mesophyll protoplasts, followed by TIDE (Tracking of Indels by DEcomposition) [42] analysis of Sanger sequence reads, and CRISPResso [43] analysis of amplicon reads. On-target activities varied substantially between gRNAs, and there was no obvious correlation between experimentally determined and in silico predicted on-target gRNA activity. Off-target mutations in homoeoalleles of EPSPS were detected at low frequencies, whereas large insertions ( $\geq 20 \mathrm{bp}$ ) of DNA vector-derived sequence were detected at surprisingly high frequencies. One of the gRNAs exhibited several properties that make it potentially suitable for the development of non-transgenic glyphosate resistant wheat.

\section{Results}

Cloning and sequencing of EPSPS in wheat cv. Fielder

A vast number of intervarietal SNPs are known to exist in hexaploid wheat [44]. Therefore, for the purpose of designing effective gRNAs, we first obtained sequence information for the three homoeoalleles of EPSPS in our target wheat cv. Fielder. For each homoeoallele, two independent partial genomic clones of EPSPS were Sanger sequenced. In each case, the sequences of the two independent clones were identical. Thus, the following consensus sequences, covering a region from the $5^{\prime}$ end of intron 1 to the middle of exon 5, were obtained: TaEPSPS-FL-7AS [GenBank MG460802], TaEPSPS-FL4AL [GenBank MG460801], and TaEPSPS-FL-7DS [GenBank MG460803]. These consensus sequences mapped unambiguously to chromosomes 7AS, 4AL (translocated from 7BS) and 7DS, as expected [45]. We identified three synonymous, homoeologous SNPs located at the 3' end of exon 2, in close proximity to a universal mutation hotspot for resistance to glyphosate [46] (Fig. 1). We exploited these SNPs for the design of homoeoallele-specific gRNAs (see below).

\section{Protoplast transformation}

We designed seven gRNAs targeting a region of EPSPS that contains the universal mutation hotspot for resistance to glyphosate (Fig. 1). gRNA1, gRNA2 and gRNA7 were designed to target only one or two of the three EPSPS homoeoalleles, whereas the other four gRNAs were designed to target all three homoeoalleles. We transiently co-expressed Cas9 and each gRNA in wheat mesophyll protoplasts. To gauge transient transformation efficiencies, we used a positive control in which YFP was substituted for the gRNA. The proportion of fluorescent (YFP expressing) protoplasts in the positive control ranged from 64 to $72 \%$ (mean $=68 \%)$ (Additional file 1). We 


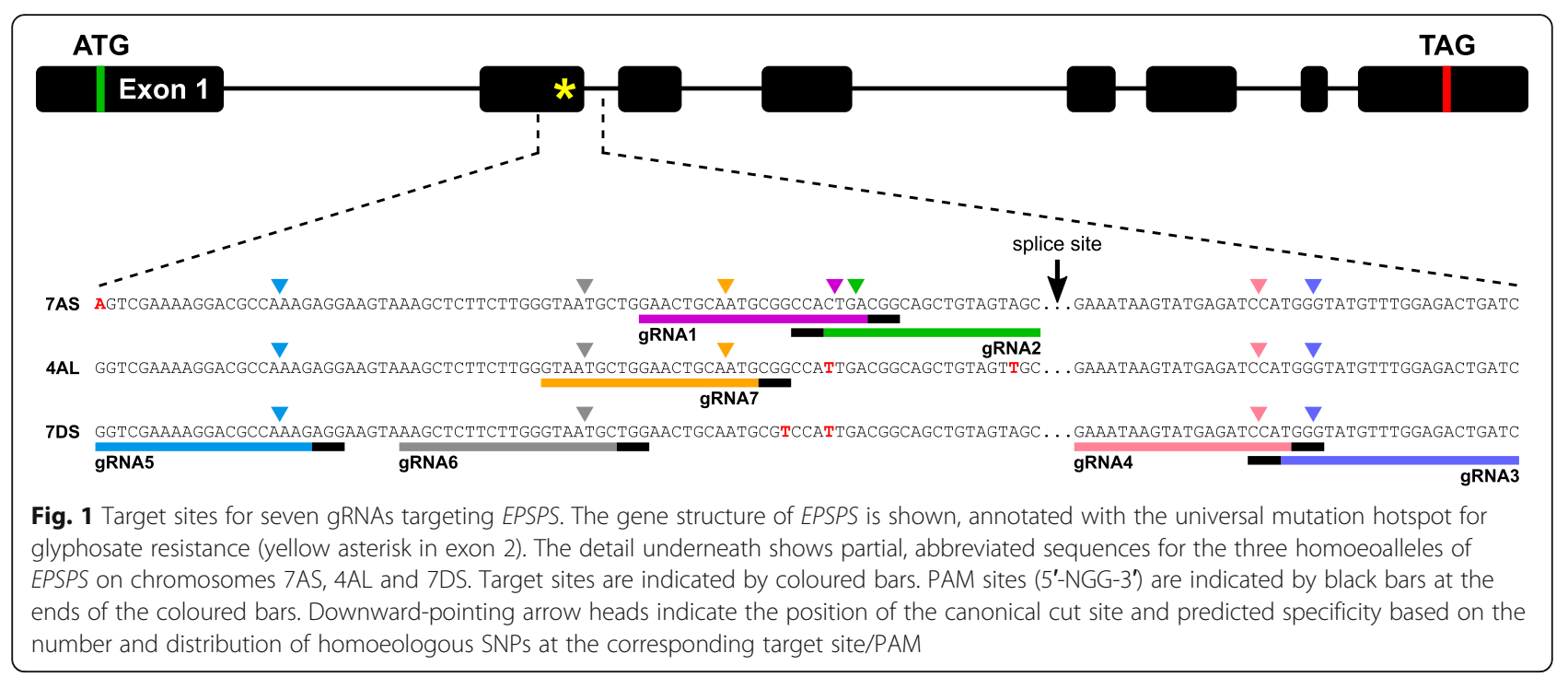

found that the key to achieving high transient transformation efficiencies was to dilute the protoplasts to a concentration of $3.0 \times 10^{5}$ cells $/ \mathrm{mL}$ (instead of $2.5 \times 10^{6}$ cells $/ \mathrm{mL}$ as described in another protocol [47]) prior to transformation, and to avoid extended incubation of DNA with protoplasts prior to adding PEG (Additional file 2).

\section{Assessment of gRNA activity and specificity via TIDE analysis of Sanger sequence traces}

We obtained high-quality forward and reverse Sanger sequence reads (Additional files 3-10) of homoeoallelespecific amplicons (Additional file 11) derived from protoplasts treated with each of the seven EPSPS-specific gRNAs and one non-targeting (random guide sequence) negative control gRNA. As expected, some of the sequence traces for samples treated with EPSPS-specific gRNAs contained mixed peaks downstream of the predicted cut site, and these mixed peaks were detected by TIDE as an increase in the percent of aberrant sequence relative to the negative control (Fig. 2a). There was a strong correlation between the indel spectra/frequencies calculated by TIDE for forward and reverse sequence traces, with each decomposition result having a high goodness of fit $\left(R^{2} \geq 0.93\right)$ (Additional file 12). Individual indels (significant at $p<0.001)$ were detected at frequencies down to approximately $1 \%$ (e.g. gRNA2, Rep 3, 7DS in Additional file 12). The mean frequency of significant indels ranged from $0.0-23.3 \%$ depending on the gRNA and homoeoallele (Fig. 2b). gRNA5 was the most highly active gRNA on all three homoeoalleles, and the presence of a single mismatch at the PAM-distal end of the guide sequence (position 20) on 7AS did not reduce the activity of gRNA5 (Fig. 2b). gRNA2 was moderately active on 7AS, and off-target indels were also detected at low frequency on 7DS in the presence of a single mismatch at the PAM-proximal end of the guide sequence (position 1) (Fig. 2b). gRNA4 was also moderately active on 7AS, but the frequency of indels appeared to be lower on 4AL and 7DS, even though no mismatches were present (Fig. 2b). All other gRNAs exhibited low or no activity (Fig. 2b).

\section{Assessment of gRNA activity and specificity via CRISPResso analysis of amplicon reads}

As an alternative method for detecting indels produced through NHEJ, and to crosscheck the TIDE results, we subjected all of the samples to amplicon deep sequencing. Due to high sequence similarity between amplicon reads derived from the three homoeoalleles of EPSPS, CRISPResso was unable to accurately map the reads to their respective reference amplicon sequences. Therefore, we used pre-mapped amplicon reads [NCBI BioProject PRJNA420019] as input for the CRISPResso analyses. In CRISPResso, the total number of aligned (analysed) amplicon reads in each subanalysis (i.e. a replicate for a gRNA on a homoeoallele) ranged from 7067 to 35,668 (mean = 18,110). In general, the CRISPResso results (Fig. 2c and d, Additional files 13 and 14) were in agreement with the TIDE results, but there was less variation between replicates in the CRISPResso results, as indicated by smaller standard errors (Fig. 2d). Notably, in the CRISPResso results, the activity of gRNA2 on 7DS (off-target) was only $7 \%$ of that on 7AS (on-target), and the difference was statistically significant $(p<0.05)$ (Fig. 2d). Also, the activity of gRNA4 was more consistent across homoeoalleles (Fig. $2 \mathrm{~d})$. The frequency of indels in the negative control was $\leq 0.1 \%$ (mean $=0.005 \%)$. 

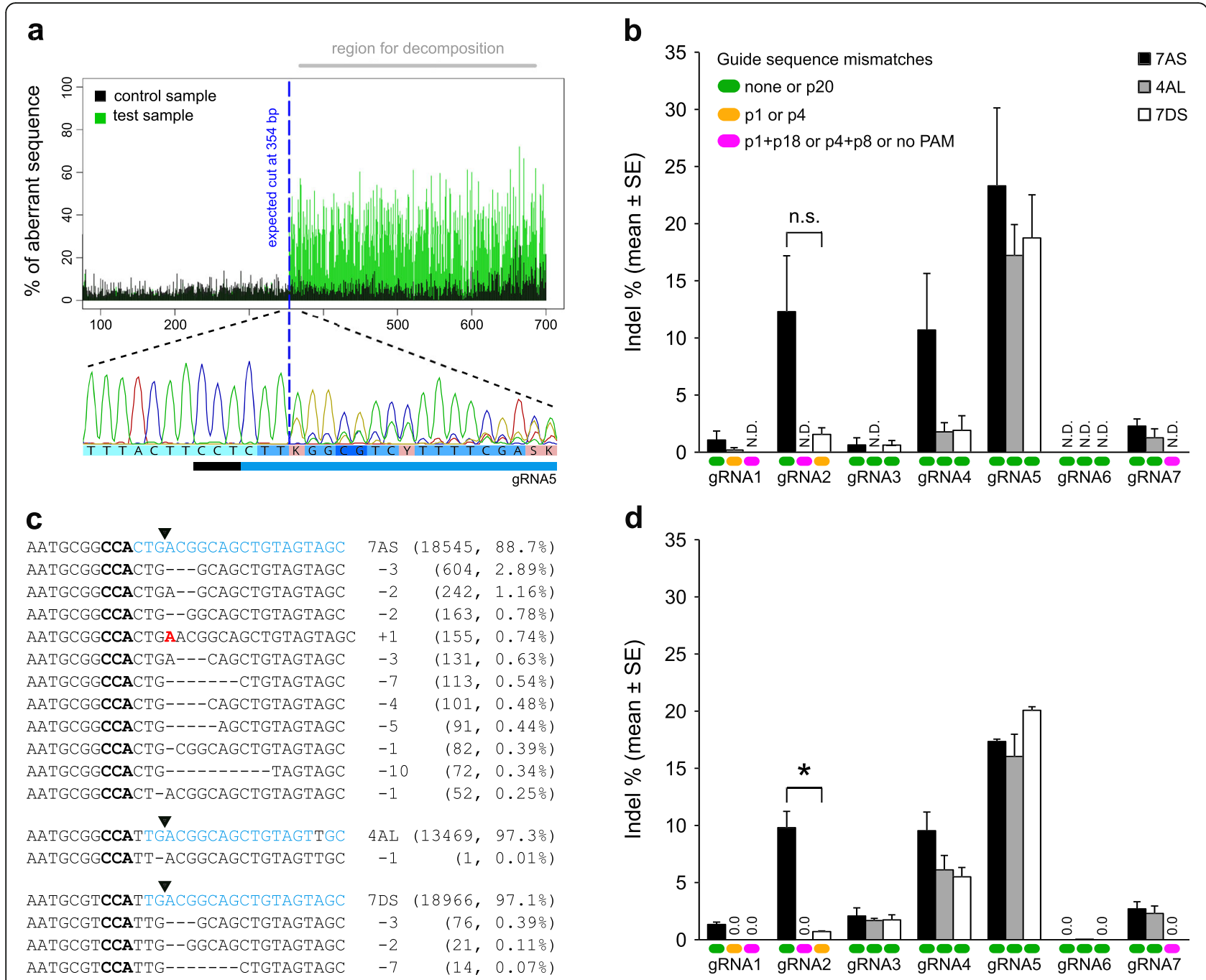

Fig. 2 Mutation detection and summary of editing efficiencies for seven gRNAs targeting EPSPS on chromosomes 7AS, 4AL and 7DS. a TIDE detection of mixed peaks in the reverse Sanger sequence read for gRNA5 on chromosome 7AS (replicate 1). b Summary of TIDE results. N.D., not detected. n.S., not statistically significant. Error bars represent the standard error of the mean $(n=3)$. c Alignment of representative mutant amplicon reads for gRNA2 on chromosomes 7AS, 4AL and 7DS (replicate 1). Bold black text, PAM; blue text, complementary to gRNA2 guide sequence; red text, inserted nucleotide. Downward-pointing arrow heads indicate the position of the canonical cut site. The number of reads and percent of total reads is shown in brackets. $\mathbf{d}$ Summary of CRISPResso results. Error bars represent the standard error of the mean $(n=3)$. * statistically significant $(p<0.05)$ based on a two-sample $t$-test assuming unequal variances. The keys in $\mathbf{b}$ also apply to $\mathbf{d}$. In the key for guide sequence mismatches, p20 means position 20 in the guide sequence, etc.

Based on both TIDE and CRISPResso derived data, we determined that gRNA2 is likely to be the most effective gRNA for generating stable homoeoallele-specific (chromosome 7AS) edits in EPSPS.

\section{In silico prediction of on-target gRNA activity}

The seven gRNAs differed substantially in their in silico predicted on-target activity (Table 1). sgRNA Designer scores [34] ranged from $0.47-0.85$ (potential range $=0-1$ ). WU-CRISPR scores [32] ranged from $<50-85$ (potential range $=0-100$; scores $<50$ are not output). There was some disagreement between the sgRNA Designer and WU-CRISPR scores. In particular, the top-scoring gRNA in sgRNA Designer (gRNA7) had a WU-CRISPR score of $<50$. There did not appear to be any obvious correlation between experimentally determined and in silico predicted on-target gRNA activity (Table 1).

\section{Analysis of large insertions}

To detect large insertions ( $\geq 20 \mathrm{bp}$ ), we used unmapped amplicon reads [NCBI BioProject PRJNA420019] as input for a separate CRISPResso analysis. Large insertions were detected in the majority of samples. The third replicate of gRNA2 had the highest frequency of large insertions (8.5\% of edited reads), all of which showed 100\% sequence identity to components of the DNA vectors 
Table 1 Experimentally determined versus in silico predicted on-target gRNA activity

\begin{tabular}{|c|c|c|c|c|}
\hline & $\begin{array}{l}\text { Target } \\
\text { homoeoalleles }\end{array}$ & $\begin{array}{l}\text { Indel } \\
\text { frequency } \\
(\%)^{\mathrm{a}}\end{array}$ & $\begin{array}{l}\text { sgRNA Designer } \\
\text { score }^{b}\end{array}$ & $\begin{array}{l}\text { WU-CRISPR } \\
\text { score }^{c}\end{array}$ \\
\hline gRNA1 & 7AS & 1.3 & 0.59 & 58 \\
\hline gRNA2 & 7AS & 9.8 & 0.58 & 60 \\
\hline gRNA3 & 7AS, 4AL, 7DS & 1.8 & 0.64 & 82 \\
\hline gRNA4 & $7 A S, 4 A L, 7 D S$ & 7.0 & 0.52 & $<50$ \\
\hline gRNA5 & $7 A S, 4 A L, 7 D S$ & 17.8 & 0.68 & 85 \\
\hline gRNA6 & 7AS, 4AL, 7DS & 0.0 & 0.47 & $<50$ \\
\hline gRNA7 & $7 A S, 4 A L$ & 2.5 & 0.85 & $<50$ \\
\hline
\end{tabular}

${ }^{a}$ Experimentally determined on-target gRNA activity, expressed as the mean proportion of edited amplicon reads derived from target homoeoalleles for three replicates. ${ }^{\mathrm{b}}$ Potential range $=0-1$ ( 1 is highest predicted gRNA activity); $\mathbf{c}$ potential range $=0-100$ (100 is highest predicted gRNA activity, scores $<50$ are not output)

used for protoplast transformation (Fig. 3, Additional file 15). Similar frequencies of large insertions were observed for the third replicate of gRNA5 (5.8\% of edited reads), and no large insertions were detected in the negative controls (Additional file 15). CRISPResso failed to correctly predict the size of the insertion when the insertion was accompanied by a deletion. For example, the $+42 /-31$ and $+54 /-1$ mutations (Fig. 3) were listed as +21 and +53 insertions respectively, in the CRISPResso allele frequency table (Additional file 15).

\section{Discussion}

We assessed the activity and specificity of seven gRNAs targeting EPSPS in wheat mesophyll protoplasts. Certain amino acid substitutions in EPSPS are known to confer resistance to the herbicide glyphosate [38], and therefore EPSPS is an ideal candidate for editing via HDR given that glyphosate resistance is a robust selectable marker in wheat tissue culture and during plant growth [48, 49]. Furthermore, in wheat it may be possible to avoid the severe yield penalty associated with homozygous amino acid substitutions and loss-of-function mutations in EPSPS [39-41], by performing homoeoallele-specific editing. However, given the inherent low frequency of HDR, and the potential for simultaneous editing of all three homoeoalleles, this application requires the use of a highly active and (ideally) homoeoallele-specific gRNA. With this in mind, our aim was to identify such a gRNA, and in doing so build on previous work [47] to develop an improved method for validating gRNAs in wheat and other polyploid species.

The wide range of on-target activities observed for the tested gRNAs in this study is consistent with previous reports of CRISPR-based genome editing using hexaploid wheat mesophyll protoplasts. A gRNA targeting TaMLO-A1 caused indels at a frequency of $29 \%$ based on the PCR-restriction enzyme (PCR-RE) assay [9]. In a separate study, the same gRNA caused indels at a frequency of $36 \%$ based on a homoeoallele-specific T7E1 assay [50]. Similar editing efficiencies were attained with gRNAs targeting TaGW2 and TaGASR7 [51]. gRNAs targeting TaDEP1, TaNAC2, TaPIN1 and TaLOX2 were also evaluated by the PCR-RE assay [20], but the editing efficiencies were not calculated. Nevertheless, it could be seen that editing efficiencies varied substantially between gRNAs and were comparable with those presented here

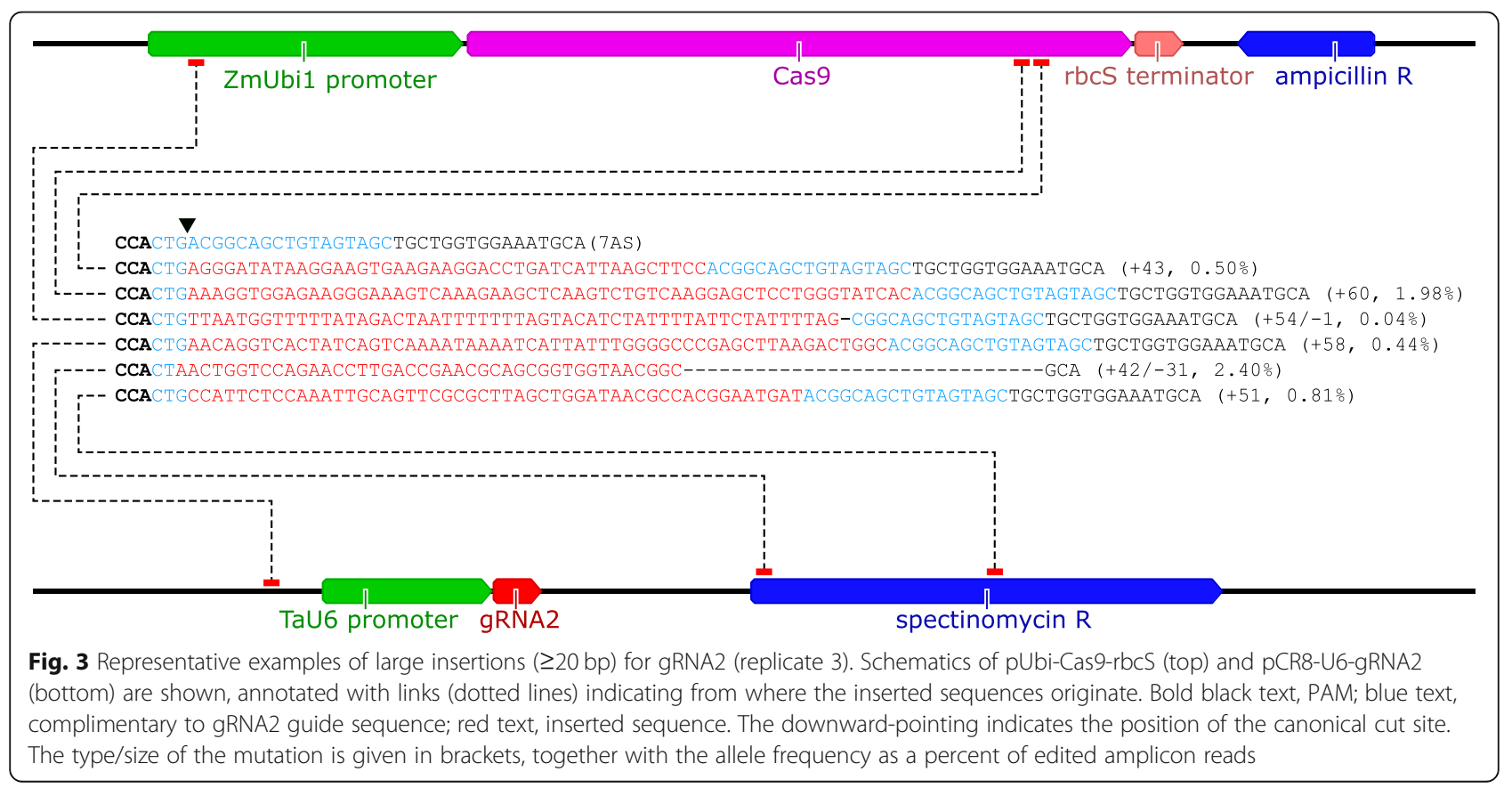


for EPSPS. gRNAs targeting TaDREB2 and TaERF3 caused indels at a frequency of 6.7 and $10.2 \%$ respectively, based on the T7E1 assay [52]. It has been reported that approximately three or four gRNAs out of ten induce indels at a frequency of $>20 \%$ in wheat protoplasts (whereas in rice protoplasts the number is approximately seven or eight gRNAs out of ten) [47]. Out of seven gRNAs, we found that one induced indels at a frequency of almost $20 \%$, two induced indels at a frequency of $7-$ $10 \%$, and four induced indels at a frequency of $<3 \%$ (based on data presented in Table 1). Taken together, this limited dataset suggests that gRNAs with high activity in wheat are likely the exception rather than the rule. Moreover, we did not find any obvious correlation between experimentally determined and in silico predicted on-target gRNA activity, which suggests that further improvements to bioinformatics tools for gRNA design are needed. For these reasons, we consider it prudent to carry out gRNA validation prior to commencing experiments for the production of stably edited wheat plants.

We observed low levels of gRNA activity at off-target homoeoalleles of EPSPS. Our results are consistent with established models of gRNA specificity [27-30] in which: a) the absence of a canonical PAM site (5'-NGG-3') greatly reduces or abolishes gRNA activity; b) mismatches within the PAM-proximal 8-12 nucleotide seed region of the guide sequence reduce gRNA activity to a greater degree than mismatches outside the seed region; and c) additional mismatches further reduce gRNA activity. Importantly, a single mismatch within the seed region (at position 4 in gRNA1 and position 1 in gRNA2) greatly reduced but did not abolish gRNA activity. However, when the mismatch within the seed region was accompanied by another mismatch (at position 8 in gRNA1 and position 18 in gRNA2), gRNA activity was further reduced to levels that were undetectable with Sanger sequencing and barely detectable with amplicon deep sequencing. These results are consistent with previous reports, which showed that off-target mutations can occur in plants when there is only a single mismatch in the seed region [20, 25]. In these studies, frequencies of off-target mutagenesis were approximately $50-80 \%$ lower than frequencies of on-target mutagenesis [20, 25]. By contrast, the gRNA targeting TaMLO-A1 (mentioned above) did not appear to generate any off-target mutations in homoeoalleles (TaMLO-B1 and TaMLO$D 1$ ) in wheat protoplasts or transgenic $\mathrm{T}_{0}$ plants, due to the presence of a single mismatch at position 2 [50]. The apparent greater reduction in gRNA activity in the presence of a mismatch at position 1,2 or 4 (compared with a mismatch at position 7,8 or 9) may be due to the existence of five nucleotide 'core' within the seed region at the PAM-proximal end of the guide sequence $[29,30]$. These results suggest that although off-target mutations are substantially reduced in the presence of a single mismatch in the seed region, they are often not eliminated. Therefore, ideally, potential off-target sites should lack a PAM, or else contain multiple mismatches, including at least one in the core of the seed region [24]. Where this is not possible, higher specificity may be achieved through the use of a truncated gRNA [53] and/or high fidelity variant of Cas9 [54]. Surprisingly, the activity of gRNA4 was apparently reduced on 4AL and 7DS, even though no mismatches were present. The reason for this is unknown. However, given that the reductions were less pronounced in the amplicon deep sequencing data (Fig. 2d), it would seem that this unexpected result is at least partly explained by the PCR and/or sequencing method used.

gRNA2 exhibited several properties that make it potentially suitable for the development of non-transgenic glyphosate resistant wheat. First, the canonical cut site for gRNA2 is adjacent to the universal mutation hotspot for resistance to glyphosate. This is important because the frequency of HDR tends to decrease as the distance between the DSB and the site of the desired mutation increases [55]. Second, gRNA2 was active at its target site on 7AS, although the activity was moderate. Third, gRNA2 was relatively specific for EPSPS on 7AS, which is the most highly transcribed copy of EPSPS in at least some wheat cultivars [45]. This high specificity would facilitate the creation of an EPSPS mutant that is edited on 7AS and wild type on 4AL/7DS. Such a mutant would have the desired trait (glyphosate resistance), and the yield penalty that could otherwise result from simultaneous modification or knockout of EPSPS on 4AL/7DS would be avoided.

One somewhat surprising finding in this study was the relatively high frequency of insertions (up to $8.5 \%$ of edited amplicon reads) that show $100 \%$ sequence identity to components of the DNA vectors used for transformation. These insertions are almost certainly vector-derived, and some (e.g. synthetic Cas9 sequences) are undoubtedly vector-derived. Recently, it was reported that DNA vector-derived insertions occur at very low frequencies (0.06-0.14\% of edited amplicon reads) in Arabidopsis protoplasts transiently transformed with CRISPR-Cas9 vectors [56], although the authors state that the frequencies were likely underestimated because insertions of $>50$ bp were excluded from the analysis. In addition to experimental differences, species-specific differences in NHEJ [57] may help to explain the much higher frequencies of DNA vector-derived insertions in wheat. If so, then sequence knockin via MMEJ may be a particularly effective genome editing strategy in wheat $[19,50]$. On a related note, if DNA vector-derived sequences were to be found in an unregulated genome-edited crop (i.e. at an uncharacterised off-target site), it would set back both the 
regulatory authority and the industry [56]. Fortunately, recent advances in crop genome editing with in vitro transcribed RNA [20] and Cas9 ribonucleoproteins [51, 58, 59] suggest that the potential for unintended insertions of DNA vector-derived sequences can be eliminated in many cases. Where these methods cannot be used, unwanted DNA-vector derived insertions could be detected by whole genome sequencing and, if not linked to the target modification, removed by backcrossing.

Finally, this study demonstrates the utility of two methods for detecting and quantifying on-target and offtarget indels: TIDE analysis of Sanger sequence traces, and CRISPResso analysis of amplicon reads. Although restriction enzyme-based assays such as PCR-RE and T7E1 are rapid, cheap and widely used for mutation detection, they suffer from several drawbacks and may not be suitable in some circumstances. For example, if the target site lacks a restriction site at the canonical cut site (as was the case with gRNA6 in this study), then the PCR-RE assay is impractical. On the other hand, the T7E1 enzyme recognises and cleaves mismatched DNA produced through denaturation and re-annealing of wild type and mutant PCR amplicons, so it does not require a restriction site. However, the T7E1 assay may produce results that are difficult to interpret if the wild type amplicons are polymorphic (a potential problem in polyploids) [60]. Moreover, restriction enzyme-based assays provide essentially no information about the indel spectrum or sequences of mutant alleles. Such information can be useful for the purpose of selecting gRNAs for particular applications [61, 62]. The sequencing-based mutation detection methods used in this study overcome the limitations described above, and come with the added benefit of greater sensitivity. Sanger sequencing combined with TIDE analysis takes only a few days and can be costeffective for low/medium throughput screening, considering that sequencing of the opposite strand is unnecessary if the forward sequence trace is high quality. Amplicon deep sequencing combined with CRISPResso analysis takes longer and is only cost-effective for high throughput screening. In some cases, significant expertise in bioinformatics may be required for the analysis of amplicon reads derived from polyploid species. A number of other mutation detection methods have been established [63, 64]. Ultimately, the choice of mutation detection method should be made on a case-by-case basis, taking into consideration the goals of the experiment and the available resources [60].

\section{Conclusions}

In summary, this study demonstrates that gRNA validation is an essential step in the application of the CRISPR-Cas9 system in wheat. gRNA validation should be carried out prior to commencing transformation and tissue culture experiments for the production of stably edited wheat plants. We have established a rapid and reliable method for assessing gRNA activity and specificity in hexaploid wheat. The method is based on an improved wheat protoplast transformation protocol, as well as the use of sequencing-based mutation detection techniques that overcome many of the limitations of commonly used enzyme-based assays. The method was used to identify a gRNA that could potentially be used for the production of non-transgenic glyphosate resistant wheat lines. Our approach is applicable to any plant species amenable to protoplast transformation, and should facilitate the adoption of CRISPR-Cas9 technology for genome editing in wheat and other polyploid crops.

\section{Methods}

Cloning and sequencing of EPSPS in wheat cv. Fielder

A full-length Triticum aestivum EPSPS cDNA consensus sequence (1789 bp) was retrieved from GenBank [EU977181] and used as the query for a BLASTN search against the $T$. aestivum EST database. The returned ESTs were assembled de novo into contigs using the Geneious Assembler in Geneious v9. The cDNA consensus sequence and EST-derived contigs were then aligned with genomic sequences from the TGACv1 wheat genome assembly (scaffold_569503_7AS:9611-10,115, scaffold_290435_4AL:41770-42,544 and scaffold_623048_ 7DS:39649-41,774), using the MUSCLE Alignment tool in Geneious. Based on this multiple sequence alignment, primers were designed (Additional file 16) to amplify a > $2 \mathrm{~kb}$ region of the three homoeologous copies of EPSPS in cv. Fielder. Amplicons were TOPO cloned into pCR8 (Invitrogen), and two independent pCR8-TaEPSPS-FL clones derived from each homoeoallele (based on diagnostic restriction enzyme digest) were validated by Sanger sequencing (Australian Genome Research Facility). The Sanger sequence reads were aligned to produce a consensus sequence for each homoeoallele. The consensus sequences were then incorporated into the multiple sequence alignment and used as the basis for gRNA design.

\section{gRNA design}

Seven gRNAs were manually designed to target EPSPS. Target sites were 20-22 nucleotides in length, and were located immediately $5^{\prime}$ of a PAM sequence (5'-NGG$\left.3^{\prime}\right)$. An extra $G$ nucleotide was appended to the $5^{\prime}$ end of gRNA6 in order to ensure efficient transcription of the gRNA expressed under the U6 promoter [65].

\section{In silico prediction of on-target gRNA activity}

On-target gRNA activity was predicted using the sgRNA Designer [34, 66] and WU-CRISPR [32, 67] tools, according to the developers' guidelines. 


\section{Vector design and construction}

All vectors were designed using Geneious software. To construct the gRNA vector, the gRNA expression cassette [9] consisting of the TaU6 promoter and a non-targeting gRNA was synthesised (GenScript) and TOPO cloned into pCR8 (Invitrogen). The BbsI site in the pCR8 backbone was then removed by digestion with NheI and selfligation, resulting in pCR8-U6-NCgRNA (negative control for editing). To insert guide sequences into pCR8-U6NCgRNA, the guide sequence oligos (Additional file 16) were first annealed by combining $1 \mu \mathrm{L}$ of each oligo $(100 \mu \mathrm{M})$ with $1 \mathrm{X}$ T4 DNA ligase buffer (Invitrogen) in a total reaction volume of $10 \mu \mathrm{L}$. The reaction was heated to $95^{\circ} \mathrm{C}$ for $5 \mathrm{~min}$ and then left at room temperature for 30 min. Annealed oligos were inserted into pCR8-U6NCgRNA by simultaneous digestion/ligation using $1 \mu \mathrm{L}$ annealed oligos, $50 \mathrm{ng}$ pCR8-U6-NCgRNA, 1X NEBuffer 2.1, 2 units BbsI (New England Biolabs), 1X T4 DNA ligase buffer, and 0.5 units T4 DNA ligase (Invitrogen) in a total reaction volume of $10 \mu \mathrm{L}$. Cycling conditions were as follows: $37^{\circ} \mathrm{C}$ for $1 \mathrm{~h}, 15^{\circ} \mathrm{C}$ for $1 \mathrm{~min}, 20^{\circ} \mathrm{C}$ for $10 \mathrm{~min}(2$ cycles), and finally $37^{\circ} \mathrm{C}$ for $1 \mathrm{~h}$. Positive clones of pCR8U6-gRNA1/2/3/4/5/6/7 were identified by diagnostic double digest with BbsI and EcoRI-HF (New England Biolabs), and validated by Sanger sequencing (Australian Genome Research Facility).

To construct the Cas9 vector, the rice codon-optimised SpCas9 gene with $\mathrm{N}$ - and C-terminal nuclear localisation signals [9] was synthesised (GenScript) and inserted into the generic vector pUbi-rbcS as an NcoI-AscI fragment between the maize Ubiquitin 1 promoter $[68,69]$ and the wheat rbcS Class II terminator [70], resulting in pUbiCas9-rbcS.

To construct the YFP vector, the EYFP gene was inserted into pUbi-rbcS in the same manner as above, resulting in pUbi-YFP-rbcS.

\section{Protoplast isolation and transformation}

Protoplast isolation and transformation was carried out as described [47], with several modifications. Seedlings of $T$. aestivum cv. Fielder were grown in potted soil within a growth chamber at $24^{\circ} \mathrm{C}$ with a photoperiod of $12 \mathrm{~h}$ light $\left(\sim 100 \mu \mathrm{mol} \mathrm{m}^{-2} \mathrm{~s}^{-1}\right)$ and $12 \mathrm{~h}$ dark, for $7-8$ days. Only vigorous seedlings (five to eight in total) were used for protoplast isolation. A razor blade was used to make a shallow cut across the adaxial surface of the primary leaf, from which the abaxial epidermis was peeled off. Leaf peels were placed abaxial side down in a petri dish containing $0.6 \mathrm{M}$ mannitol for $15 \mathrm{~min}$. Leaf peels were then placed abaxial side down in a petri dish containing $10 \mathrm{~mL}$ of cell wall-dissolving enzyme solution [20 mM MES-KOH (pH 5.7), 1.5\% (wt/vol) cellulase Onozuka RS, 0.75\% (wt/vol) macerozyme R10, 0.6 M mannitol, $10 \mathrm{mM} \mathrm{KCl}, 10 \mathrm{mM} \mathrm{CaCl}, 0.1 \%$ (wt/vol) BSA] for $3-4 \mathrm{~h}$ with very gentle agitation. After addition of one volume of W5 solution [2 mM MES-KOH ( $\mathrm{pH}$ 5.7), $154 \mathrm{mM} \mathrm{NaCl}, 125 \mathrm{mM} \mathrm{CaCl}, 5 \mathrm{mM} \mathrm{KCl}$ [71], protoplasts were filtered through a $100 \mu \mathrm{m}$ nylon mesh into a petri dish and then carefully transferred to a $30 \mathrm{~mL}$ round-bottom tube (Sarstedt 55.517). Protoplasts were centrifuged for $3 \mathrm{~min}$ at $80 \mathrm{xg}$, resuspended in $15 \mathrm{~mL}$ of W5 solution, and incubated on ice for $30 \mathrm{~min}$. The W5 solution was removed, and the protoplasts were resuspended in $500 \mu \mathrm{L}$ MMG solution [ $4 \mathrm{mM}$ MES- $\mathrm{KOH}$ (pH 5.7), $0.4 \mathrm{M}$ mannitol, $15 \mathrm{mM} \mathrm{MgCl}_{2}$ ] [71]. The protoplast concentration was determined by cell counting on a hemocytometer, and subsequently adjusted to $3.0 \times 10^{5}$ cells $/ \mathrm{mL}$ using MMG solution.

In an empty $2 \mathrm{~mL}$ tube, Ubi-Cas9-rbcS $(20 \mu \mathrm{g}, 3.5$ pmol) was mixed with either pCR8-U6-gRNA1/2/3/4/5/ 6/7 (gRNAs targeting EPSPS) (20 $\mu \mathrm{g}, 10.5 \mathrm{pmol}), \mathrm{pCR} 8$ U6-NCgRNA (negative control for editing) $(20 \mu \mathrm{g}, 10.5$ pmol), or pUbi-YFP-rbcS (positive control for transformation) $(20 \mu \mathrm{g}, 5.7 \mathrm{pmol})$. Transformation was carried out by adding (in quick succession) $200 \mu \mathrm{L}$ of protoplasts and then $200 \mu \mathrm{L}$ of PEG solution [40\% (wt/vol) PEG4000, $0.2 \mathrm{M}$ mannitol, $100 \mathrm{mM} \mathrm{CaCl}_{2}$ ] to the tube containing pre-mixed DNA. The DNA/protoplast/PEG mixture was homogenised by gently flicking the tube, and then incubated for $15 \mathrm{~min}$ at room temperature. The transformation reaction was stopped by adding $840 \mu \mathrm{L}$ of W5 solution and gently inverting the tube three times. The protoplasts were centrifuged for $2 \mathrm{~min}$ at $100 \times g$. The supernatant was removed and the protoplasts were resuspended in $500 \mu \mathrm{L} \mathrm{W5}$ solution. The protoplasts were then transferred to 12-well plates (Sarstedt 83.3921 .500 ) coated with $5 \% \mathrm{vol} / \mathrm{vol}$ fetal bovine serum (Sigma-Aldrich F4135), and incubated at $23^{\circ} \mathrm{C}$ in the dark for $48 \mathrm{~h}$.

The experiment was repeated twice more, from the seed planting step. Thus, there were three biological replicates for each treatment and control.

\section{Microscopy}

After 16-24h of incubation, protoplasts co-transformed with pUbi-Cas9-rbcS and pUbi-YFP-rbcS (positive control for transformation) were imaged using a Nikon Ni-E microscope equipped with a $490-500 \mathrm{~nm}$ excitation filter and a $520-560 \mathrm{~nm}$ emission filter (Adelaide Microscopy Waite Facility). Transformation efficiencies were calculated as the proportion of spherical protoplasts $(n=100$, bright field image) that emitted yellow fluorescence (dark field image).

\section{Flow cytometry}

After $20 \mathrm{~h}$ of incubation, protoplasts co-transformed with pUbi-Cas9-rbcS and pUbi-YFP-rbcS were subjected 
to flow cytometry using a BD Accuri C6. For the negative control, water was used instead of DNA.

\section{gDNA extraction}

At the end of the $48 \mathrm{~h}$ incubation period, protoplasts were transferred to $2 \mathrm{~mL}$ tubes and centrifuged for 2 $\min$ at $100 \times g$. The supernatant was removed and gDNA was extracted from the protoplast pellet using the DNeasy Plant Mini Kit (QIAGEN) according to manufacturer's instructions. DNA was eluted from the spin column with $28 \mu \mathrm{L}$ of elution buffer.

\section{Sanger sequencing and TIDE analysis}

To obtain amplicons for Sanger sequencing, a genomic region (1781 bp on 7AS, $1572 \mathrm{bp}$ on 4AL, and $1701 \mathrm{bp}$ on 7DS) containing all seven target sites was amplified by PCR using homoeoallele-specific primers (Additional file 16). PCR was performed using 30-40 ng gDNA template, $0.8 \mu \mathrm{M}$ primers, $200 \mu \mathrm{M}$ dNTPs, $1 \mathrm{X}$ Phusion HF buffer, and 0.6 units Phusion Hot Start Flex DNA Polymerase (New England Biolabs) in a total reaction volume of $50 \mu \mathrm{L}$. gDNA obtained from nulli-tetrasomic lines of $T$. aestivum cv. Chinese Spring was used as template in control PCR reactions to confirm that amplification was homoeoallele-specific. Cycling conditions for touchdown PCR were as follows: initial denaturation at $98^{\circ} \mathrm{C}$ for 1 min, denaturation at $98^{\circ} \mathrm{C}$ for $5 \mathrm{~s}$, annealing at $68-63^{\circ} \mathrm{C}$ (7AS and 7DS) or $66-61{ }^{\circ} \mathrm{C}(4 \mathrm{AL})$ for $15 \mathrm{~s}$, extension at $72{ }^{\circ} \mathrm{C}$ for $55 \mathrm{~s}$, and final extension at $72^{\circ} \mathrm{C}$ for $5 \mathrm{~min}$. The starting annealing temperature was decreased by $0.5{ }^{\circ} \mathrm{C}$ each cycle for 10 cycles, followed by 30 cycles at the final annealing temperature. The PCR product was run on $1 \%$ agarose gel, from which amplicons were extracted using the NucleoSpin Gel and PCR Clean-up kit (MachereyNagel) according to the manufacturer's instructions. DNA was eluted from the spin column with $15 \mu \mathrm{L}$ of diluted (1 in 40) elution buffer and quantified using a NanoDrop 1000 spectrophotometer.

To detect targeted indels produced via NHEJ, homoeoallele-specific amplicons from each PCR reaction were subjected to Sanger sequencing (Australian Genome Research Facility) in the forward and reverse directions with nested homoeoallele-specific primers (Additional file 16). The 3730xl DNA Analyzer (Applied Biosystems) was used for sequencing, and bases were called with KB Basecaller v1.4.1.8. Output AB1 files for treated and untreated (negative control) samples were uploaded to the online TIDE analysis tool [42]. In TIDE, minor adjustments to the decomposition window were made based on information provided on the online TIDE analysis tool Troubleshooting webpage. All other TIDE settings were the default. The indel frequency for each gRNA/homoeoallele/replicate was calculated as the mean percent of sequences containing significant indels $(p<0.001)$ for the forward and reverse reads.

\section{Amplicon deep sequencing and CRISPResso analysis}

To obtain amplicons for deep sequencing, two rounds of PCR were carried out. In the first round of PCR, a genomic region (269 bp on $7 \mathrm{AS}$, and $270 \mathrm{bp}$ on $4 \mathrm{AL} / 7 \mathrm{DS}$ ) containing all seven target sites was amplified using conserved primers containing $5^{\prime}$ universal tail sequences (Additional file 16) to which Illumina index primers anneal in the second round of PCR. PCR was performed using $20-40 \mathrm{ng}$ gDNA template, $0.25 \mu \mathrm{M}$ primers, $200 \mu \mathrm{M}$ dNTPs, 1X Phusion HF buffer, and 0.2 units Phusion Hot Start Flex DNA Polymerase in a total reaction volume of $20 \mu \mathrm{L}$. Cycling conditions for touchdown PCR were as follows: initial denaturation at $98^{\circ} \mathrm{C}$ for 1 min, denaturation at $98^{\circ} \mathrm{C}$ for $5 \mathrm{~s}$, annealing at $62-57^{\circ} \mathrm{C}$ for $15 \mathrm{~s}$, extension at $72{ }^{\circ} \mathrm{C}$ for $10 \mathrm{~s}$, and final extension at $72^{\circ} \mathrm{C}$ for $2 \mathrm{~min}$. The starting annealing temperature was decreased by $0.5^{\circ} \mathrm{C}$ each cycle for 10 cycles, followed by 25 cycles at the final annealing temperature. The PCR product was purified using Agencourt AMPure XP beads (Beckman Coulter) according to the manufacturer's instructions. The second round of PCR was performed using $10 \mathrm{ng}$ DNA template (purified amplicons from the first round of PCR), $0.3 \mu \mathrm{M}$ primers (Illumina Nextera XT), $200 \mu \mathrm{M}$ dNTPs, 1X Phusion HF buffer, and 0.2 units Phusion Hot Start Flex DNA Polymerase in a total reaction volume of $10 \mu \mathrm{L}$. Cycling conditions were as follows: initial denaturation at $98^{\circ} \mathrm{C}$ for $1 \mathrm{~min}$, denaturation at $98^{\circ} \mathrm{C}$ for $5 \mathrm{~s}$, annealing at $60^{\circ} \mathrm{C}$ for $15 \mathrm{~s}$, extension at $72^{\circ} \mathrm{C}$ for $6 \mathrm{~s}$, and final extension at $72{ }^{\circ} \mathrm{C}$ for $2 \mathrm{~min}$ ( 7 cycles in total). The indexed PCR products were purified using Agencourt AMPure XP beads.

Indexed PCR products were quantified by $\mathrm{qPCR}$, diluted to $4 \mathrm{nM}$, pooled in equal volumes, spiked with $10 \%$ PhiX Control v3, and then sequenced on the Illumina MiSeq platform using the MiSeq Reagent Kit v3 600 cycle (Australian Genome Research Facility). The 300 bp unpaired raw reads from each sample were mapped to the three homoeologous amplicon reference sequences in two phases using Bowtie 2 [72]. The aim of the first phase was to map unedited reads using the following parameters: --end-to-end --very-sensitive --np 0 --mp 6, 1 --rdg 999,999 --rfg 999,999 --score-min L,-6,0. Unmapped reads from the first phase were used as input for the second phase, where reads with indels (deletions of up to $51 \mathrm{bp}$ or insertions of up to $4 \mathrm{bp}$ ) and some low quality mismatches were mapped using the following parameters: --end-to-end --very-sensitive --np 0 --mp 76,1 --rdg 24,1 --rfg 9,14 --score-min L,-75,0. Next, the resulting two BAM files were sliced for the reads that mapped to the respective three amplicons and merged together using SAMtools [73]. An in-house bash script 
was used to extract the mapped unedited/edited reads from the merged BAM files, and these sequence files in FASTQ format were used as input for CRISPResso [43] analysis using the following parameters: --w 20 --hide mutations_outside_window_NHEJ --save_also_png --trim sequences -q 30 --exclude_bp_from_left 5 --exclude_bp_ from_right 5 --ignore_substitutions. Allele frequencies shown in Fig. 2c were calculated by summing the values in the \%Reads column of the CRISPResso allele frequency table, after applying an Excel text filter (Custom AutoFilter) to only show rows where the aligned sequence contains the allele sequence.. For each of the three biological replicates, indel frequencies were calculated as the \% NHEJ for the gRNA minus the \%NHEJ for the negative control, based on data from the CRISPResso pie charts.

\section{Analysis of large insertions}

To detect large insertions ( $\geq 20 \mathrm{bp}$ ), a separate CRISPResso analysis was carried out using unmapped amplicon deep sequencing reads as input, with the same CRISPResso settings as above. Data in CRISPResso allele frequency tables were sorted based on insertion size (largest to smallest), and then filtered to exclude aligned sequences containing insertions of $<20 \mathrm{bp}$. Reads containing insertions of $\geq 20$ bp were aligned to the cv. Fielder consensus sequences in Geneious using the MUSCLE alignment tool. The sequences of the insertions were then searched for in the sequences of pUbi-Cas9-rbcS and pCR8-U6-gRNA2. Allele frequencies shown in Fig. 3 were calculated by summing the values in the \%Reads column of the CRISPResso allele frequency table, after applying an Excel text filter (Custom AutoFilter) to only show rows where the aligned sequence contains the allele sequence.

\section{Supplementary information}

Supplementary information accompanies this paper at https://doi.org/10. 1186/s12896-019-0565-z.

Additional file 1. Bright field and dark field microscopy of protoplasts co-transformed with pUbi-Cas9-rbcS and pUbi-YFP-rbcS (A, C, E, G, I, K), and untransformed protoplasts (B, D, F, H, J, L). Three replicates (A-D, E-H, and $\mathrm{I}-\mathrm{L}$ ) for each treatment are shown, along with the transformation efficiency for each replicate (\% of protoplasts transformed). Scale bar = $100 \mu \mathrm{m}$.

Additional file 2. Flow cytometry of protoplasts co-transformed with pUbi-Cas9-rbcS and pUbi-YFP-rbcS. Protoplasts were diluted to different concentrations (50,000-500,000 cells per $200 \mu \mathrm{L}$ ) for transformation, and either incubated with the DNA for 10 min prior to the addition of PEG or not incubated with the DNA (PEG added immediately to DNA/protoplast mixture). The percent of protoplasts expressing YFP is indicated. MFI, mean fluorescence intensity of YFP-expressing protoplasts.

Additional file 3. Forward and reverse Sanger sequence reads of homoeoallele-specific amplicons derived from protoplasts treated with gRNA1.

Additional file 4. Forward and reverse Sanger sequence reads of homoeoallele-specific amplicons derived from protoplasts treated with gRNA2.
Additional file 5. Forward and reverse Sanger sequence reads of homoeoallele-specific amplicons derived from protoplasts treated with gRNA3.

Additional file 6. Forward and reverse Sanger sequence reads of homoeoallele-specific amplicons derived from protoplasts treated with gRNA4.

Additional file 7. Forward and reverse Sanger sequence reads of homoeoallele-specific amplicons derived from protoplasts treated with gRNA5.

Additional file 8. Forward and reverse Sanger sequence reads of homoeoallele-specific amplicons derived from protoplasts treated with gRNA6.

Additional file 9. Forward and reverse Sanger sequence reads of homoeoallele-specific amplicons derived from protoplasts treated with gRNA7.

Additional file 10. Forward and reverse Sanger sequence reads of homoeoallele-specific amplicons derived from protoplasts treated with a non-targeting (random guide sequence) negative control gRNA.

Additional file 11 Homoeoallele-specific amplification of EPSPS on chromosomes 7AS, 4AL and 7DS following transient co-expression of Cas9 and gRNA in wheat protoplasts. Nulli-tetra (-) genomic DNA template does not contain the target chromosome. Nulli-tetra (+) genomic DNA template does contain the target chromosome. NT, no template; *gRNA, non-targeting (random guide sequence) gRNA. Three replicates were performed. All bands to the right of the ladder were gel purified and Sanger sequenced in the forward and reverse directions.

Additional file 12 TIDE indel spectra/frequencies for gRNAs 1-7 targeting EPSPS in wheat protoplasts. Shown are results for forward and reverse Sanger sequence reads of homoeoallele-specific amplicons derived from chromosomes 7AS, 4AL, and 7DS. Three replicates were performed.

Additional file 13. CRISPResso allele frequency tables used for analysis of indels induced by gRNA2 in replicate 1.

Additional file 14. CRISPResso NHEJ pie charts.

Additional file 15. CRISPResso allele frequency tables used for analysis of large insertions ( $\geq 20 \mathrm{bp}$ ) induced by gRNA2 and gRNA5 in replicate 3 .

Additional file 16. Primers and oligonucleotides used in this study.

\section{Abbreviations}

Cas9: CRISPR-associated protein 9; CRISPR: clustered regularly-interspaced short palindromic repeats; DSB: Double-strand break; EPSPS: 5enolpyruvylshikimate-3-phosphate synthase; gRNA: guide RNA: HDR: Homology directed repair; NHEJ: Non-homologous end joining; PAM: Protospacer adjacent motif; PCR-RE: PCR-restriction enzyme; SNP: Single nucleotide polymorphism; TIDE: Tracking of Indels by DEcomposition

\section{Acknowledgements}

We are grateful to Matthew Tucker (The University of Adelaide) for critical discussions and reading of the manuscript, Adam Croxford (The University of Adelaide) for providing helpful advice and technical assistance with amplicon deep sequencing, Anzu Okada (The University of Adelaide) for providing technical assistance with qPCR and amplicon deep sequencing, Gwen Mayo (Adelaide Microscopy Waite Facility) for providing microscopy assistance, and Nikolai Borisjuk (Huaiyin Normal University) for providing support in the beginning stages of the project. We are grateful for the support provided by The University of Adelaide, Australian Research Council, Grains Research and Development Corporation, and the Government of South Australia.

\section{Authors' contributions}

TA conceived the project. TA, PL, UB and RW designed the experimental work. TA carried out the experimental work and analysed the data. TA, NS, UB and NW-H designed the bioinformatics work. TA, NS and NW-H carried out the bioinformatics work. TA and NS wrote the paper. PL, UB and RW critically revised the paper. All authors read and approved the final manuscript. 


\section{Funding}

This work was funded by the Australian Centre for Plant Functional Genomics (ACPFG) Pty Ltd. The ACPFG had no role in the study other than providing financial support. TA acknowledges financial support received through an Australian Government Research Training Program Scholarship.

\section{Availability of data and materials}

The datasets supporting the conclusions of this article are included in the article and its additional files. The amplicon deep sequencing data have been deposited in the NCBI SRA repository [BioProject PRJNA420019; http:// www.ncbi.nlm.nih.gov/bioproject/420019].

\section{Ethics approval and consent to participate}

Not applicable.

\section{Consent for publication}

Not applicable.

\section{Competing interests}

The authors declare that they have no competing interests.

\section{Author details}

${ }^{1}$ Present address: CSIRO, Agriculture and Food, Canberra, ACT, Australia. ${ }^{2}$ Present address: New South Wales Department of Primary Industries, Research Excellence, Orange, NSW, Australia. ${ }^{3}$ School of Agriculture, Food \& Wine, The University of Adelaide, Waite Campus, Urrbrae, SA 5064, Australia. ${ }^{4}$ Present address: Bioinformatics Hub, School of Biological Sciences, The University of Adelaide, Adelaide, SA 5005, Australia.

\section{Received: 13 August 2018 Accepted: 30 September 2019}

\section{Published online: 30 October 2019}

\section{References}

1. Puchta H, Fauser F. Gene targeting in plants: 25 years later. Int J Dev Biol. 2013;57:629-37.

2. Jinek M, Chylinski K, Fonfara I, Hauer M, Doudna JA, Charpentier E. A programmable dual-RNA-guided DNA endonuclease in adaptive bacterial immunity. Science. 2012;337:816-21.

3. Gasiunas G, Barrangou R, Horvath P, Siksnys V. Cas9-crRNA ribonucleoprotein complex mediates specific DNA cleavage for adaptive immunity in bacteria. Proc Natl Acad Sci U S A. 2012;109:E2579-86.

4. Cong L, Ran FA, Cox D, Lin S, Barretto R, Habib N, et al. Multiplex genome engineering using CRISPR/Cas systems. Science. 2013;339:819-23.

5. Mali P, Yang L, Esvelt KM, Aach J, Guell M, DiCarlo JE, et al. RNA-guided human genome engineering via Cas9. Science. 2013;339:823-6.

6. Cho SW, Kim S, Kim JM, Kim J-S. Targeted genome engineering in human cells with the Cas9 RNA-guided endonuclease. Nat Biotechnol. 2013;31:230-2.

7. Jinek M, East A, Cheng A, Lin S, Ma E, Doudna J. RNA-programmed genome editing in human cells. Elife. 2013;2:e00471.

8. Puchta $\mathrm{H}$. The repair of double-strand breaks in plants: mechanisms and consequences for genome evolution. J Exp Bot. 2005;56:1-14.

9. Shan Q, Wang Y, Li J, Zhang Y, Chen K, Liang Z, et al. Targeted genome modification of crop plants using a CRISPR-Cas system. Nat Biotechnol. 2013;31:686-8

10. Feng Z, Zhang B, Ding W, Liu X, Yang D-L, Wei $P$, et al. Efficient genome editing in plants using a CRISPR/Cas system. Cell Res. 2013;23:1229-32.

11. Mao Y, Zhang H, Xu N, Zhang B, Gou F, Zhu J-K. Application of the CRISPRCas system for efficient genome engineering in plants. Mol Plant. 2013;6: 2008-11.

12. Feng Z, Mao Y, Xu N, Zhang B, Wei P, Yang D-L, et al. Multigeneration analysis reveals the inheritance, specificity, and patterns of CRISPR/Casinduced gene modifications in Arabidopsis. Proc Natl Acad Sci U S A. 2014; 111:4632-7.

13. Li J, Norville JE, Aach J, McCormack M, Zhang D, Bush J, et al. Multiplex and homologous recombination-mediated genome editing in Arabidopsis and Nicotiana benthamiana using guide RNA and Cas9. Nat Biotechnol. 2013;31: 688-91

14. Baltes NJ, Gil-Humanes J, Cermak T, Atkins PA, Voytas DF. DNA replicons for plant genome engineering. Plant Cell. 2014;26:151-63.
15. Svitashev S, Young JK, Schwartz C, Gao H, Falco SC, Cigan AM. Targeted mutagenesis, precise gene editing, and site-specific gene insertion in maize using Cas9 and guide RNA. Plant Physiol. 2015;169:931-45.

16. Li Z, Liu Z-B, Xing A, Moon BP, Koellhoffer JP, Huang L, et al. Cas9-guide RNA directed genome editing in soybean. Plant Physiol. 2015;169:960-70.

17. Sun $Y$, Zhang $X, W u$ C, He $Y, M a ~ Y, ~ H o u ~ H$, et al. Engineering herbicideresistant rice plants through CRISPR/Cas9-mediated homologous recombination of acetolactate synthase. Mol Plant. 2016:9:628-31.

18. Endo M, Mikami M, Toki S. Biallelic gene targeting in rice. Plant Physiol. 2016;170:667-77.

19. Nakade S, Tsubota T, Sakane Y, Kume S, Sakamoto N, Obara M, et al. Microhomology-mediated end-joining-dependent integration of donor DNA in cells and animals using TALENs and CRISPR/Cas9. Nat Commun. 2014;5:5560.

20. Zhang Y, Liang Z, Zong Y, Wang Y, Liu J, Chen K, et al. Efficient and transgene-free genome editing in wheat through transient expression of CRISPR/Cas9 DNA or RNA. Nat Commun. 2016;7:12617.

21. Andersson $M$, Turesson $H$, Nicolia A, Fält AS, Samuelsson M, Hofvander P. Efficient targeted multiallelic mutagenesis in tetraploid potato (Solanum tuberosum) by transient CRISPR-Cas9 expression in protoplasts. Plant Cell Rep. 2017:36:117-28.

22. Ryder P, McHale M, Fort A, Spillane C. Generation of stable nulliplex autopolyploid lines of Arabidopsis thaliana using CRISPR/Cas9 genome editing. Plant Cell Rep. 2017;36:1005-8.

23. Baltes NJ, Gil-Humanes J, Voytas DF. Genome engineering and agriculture: opportunities and challenges. Prog Mol Biol Transl Sci. 2017;149:1-26.

24. Cho SW, Kim S, Kim Y, Kweon J, Kim HS, Bae S, et al. Analysis of off-target effects of CRISPR/Cas-derived RNA-guided endonucleases and nickases. Genome Res. 2014:24:132-41.

25. Lawrenson T, Shorinola O, Stacey N, Li C, Østergaard L, Patron N, et al. Induction of targeted, heritable mutations in barley and Brassica oleracea using RNA-guided Cas9 nuclease. Genome Res. 2015:16:258.

26. Wolt JD, Wang K, Sashital D, Lawrence-Dill CJ. Achieving plant CRISPR targeting that limits off-target effects. Plant Genome. 2016;9. https://doi.org/ 10.3835/plantgenome2016.05.0047

27. Hsu PD, Scott DA, Weinstein JA, Ran FA, Konermann S, Agarwala V, et al. DNA targeting specificity of RNA-guided Cas9 nucleases. Nat Biotechnol. 2013;31:827-32.

28. Sternberg SH, Redding S, Jinek M, Greene EC, Doudna JA. DNA interrogation by the CRISPR RNA-guided endonuclease Cas9. Nature. 2014:507:62-7.

29. Wu X, Scott DA, Kriz AJ, Chiu AC, Hsu PD, Dadon DB, et al. Genome-wide binding of the CRISPR endonuclease Cas9 in mammalian cells. Nat Biotechnol. 2014;32:670-6.

30. O'Geen H, Henry IM, Bhakta MS, Meckler JF, Segal DJ. A genome-wide analysis of Cas9 binding specificity using ChIP-seq and targeted sequence capture. Nucleic Acids Res. 2015;43:3389-404.

31. Doench JG, Hartenian E, Graham DB, Tothova Z, Hegde M, Smith I, et al. Rational design of highly active sgRNAs for CRISPR-Cas9-mediated gene inactivation. Nat Biotechnol. 2014:32:1262-7.

32. Wong N, Liu W, Wang X. WU-CRISPR: characteristics of functional guide RNAs for the CRISPR/Cas9 system. Genome Biol. 2015;16:218.

33. Graham DB, Root DE. Resources for the design of CRISPR gene editing experiments. Genome Biol. 2015;16:260.

34. Doench JG, Fusi N, Sullender M, Hegde M, Vaimberg EW, Donovan KF, et al. Optimized sgRNA design to maximize activity and minimize off-target effects of CRISPR-Cas9. Nat Biotechnol. 2016;34:184-91.

35. Oliveros JC, Franch M, Tabas-Madrid D, San-León D, Montoliu L, Cubas P, et al. Breaking-Cas - interactive design of guide RNAs for CRISPR-Cas experiments for ENSEMBL genomes. Nucleic Acids Res. 2016;44:W267-71.

36. Liu H, Ding Y, Zhou Y, Jin W, Xie K, Chen L-L. CRISPR-P 2.0: an improved CRISPR-Cas9 tool for genome editing in plants. Mol Plant. 2017;10:530-2.

37. Haeussler M, Schönig K, Eckert H, Eschstruth A, Mianné J, Renaud J-B, et al. Evaluation of off-target and on-target scoring algorithms and integration into the guide RNA selection tool CRISPOR. Genome Biol. 2016;17:148.

38. Sammons RD, Gaines TA. Glyphosate resistance: state of knowledge. Pest Manag Sci. 2014;70:1367-77.

39. Yu Q, Jalaludin A, Han H, Chen M, Sammons RD, Powles SB. Evolution of a double amino acid substitution in the 5-enolpyruvylshikimate-3-phosphate synthase in Eleusine indica conferring high-level glyphosate resistance. Plant Physiol. 2015;167:1440-7.

40. Li J, Meng X, Zong Y, Chen K, Zhang H, Liu J, et al. Gene replacements and insertions in rice by intron targeting using CRISPR-Cas9. Nat Plants. 2016:2. 16139. 
41. Zhang HH, Zhang J, Wei P, Zhang B, Gou F, Feng Z, et al. The CRISPR/Cas9 system produces specific and homozygous targeted gene editing in rice in one generation. Plant Biotechnol J. 2014;12:797-807.

42. Brinkman EK, Chen T, Amendola M, van Steensel B. Easy quantitative assessment of genome editing by sequence trace decomposition. Nucleic Acids Res. 2014;42:e168.

43. Pinello L, Canver MC, Hoban MD, Orkin SH, Kohn DB, Bauer DE, et al. Analyzing CRISPR genome-editing experiments with CRISPResso. Nat Biotechnol. 2016:34:695-7.

44. Lai K, Lorenc MT, Lee HC, Berkman PJ, Bayer PE, Visendi P, et al. Identification and characterization of more than 4 million intervarietal SNPS across the group 7 chromosomes of bread wheat. Plant Biotechnol J. 2015; 13:97-104.

45. Aramrak A, Kidwell KK, Steber CM, Burke IC. Molecular and phylogenetic characterization of the homoeologous EPSP synthase genes of allohexaploid wheat, Triticum aestivum (L.). BMC Genomics. 2015;16:844.

46. Zhou M, Xu H, Wei X, Ye Z, Wei L, Gong W, et al. Identification of a glyphosate-resistant mutant of rice 5-enolpyruvylshikimate 3-phosphate synthase using a directed evolution strategy. Plant Physiol. 2006;140:184-95.

47. Shan Q, Wang Y, Li J, Gao C. Genome editing in rice and wheat using the CRISPR/Cas system. Nat Protoc. 2014;9:2395-410.

48. Zhou H, Arrowsmith JW, Fromm ME, Hironaka CM, Taylor ML, Rodriguez D, et al. Glyphosate-tolerant CP4 and GOX genes as a selectable marker in wheat transformation. Plant Cell Rep. 1995;15:159-63.

49. Hu T, Metz S, Chay C, Zhou HP, Biest N, Chen G, et al. Agrobacteriummediated large-scale transformation of wheat (Triticum aestivum L.) using glyphosate selection. Plant Cell Rep. 2003;21:1010-9.

50. Wang Y, Cheng X, Shan Q, Zhang Y, Liu J, Gao C, et al. Simultaneous editing of three homoeoalleles in hexaploid bread wheat confers heritable resistance to powdery mildew. Nat Biotechnol. 2014;32:947-51.

51. Liang Z, Chen K, Li T, Zhang Y, Wang Y, Zhao Q, et al. Efficient DNA-free genome editing of bread wheat using CRISPR/Cas9 ribonucleoprotein complexes. Nat Commun. 2017;8:14261.

52. Kim D, Alptekin B, Budak H. CRISPR/Cas9 genome editing in wheat. Funct Integr Genomics. 2018;18:31-41.

53. Fu Y, Sander JD, Reyon D, Cascio VM, Joung JK. Improving CRISPR-Cas nuclease specificity using truncated guide RNAs. Nat Biotechnol. 2014;32:279-84.

54. Kleinstiver BP, Pattanayak V, Prew MS, Tsai SQ, Nguyen NT, Zheng Z, et al. High-fidelity CRISPR-Cas9 nucleases with no detectable genome-wide offtarget effects. Nature. 2016;529:490-5.

55. Paquet D, Kwart D, Chen A, Sproul A, Jacob S, Teo S, et al. Efficient introduction of specific homozygous and heterozygous mutations using CRISPR/Cas9. Nature. 2016;533:125-9.

56. Kim J, Kim J-S. Bypassing GMO regulations with CRISPR gene editing. Nat Biotechnol. 2016:34:1014-5.

57. Vu GTH, Cao HX, Reiss B, Schubert I. Deletion-bias in DNA double-strand break repair differentially contributes to plant genome shrinkage. New Phytol. 2017;214:1712-21

58. Woo JW, Kim J, Kwon SI, Corvalán C, Cho SW, Kim H, et al. DNA-free genome editing in plants with preassembled CRISPR-Cas9 ribonucleoproteins. Nat Biotechnol. 2015;33:1162-4.

59. Svitashev S, Schwartz C, Lenderts B, Young JK, Cigan AM. Genome editing in maize directed by CRISPR-Cas9 ribonucleoprotein complexes. Nat Commun. 2016;7:13274.

60. Zischewski J, Fischer R, Bortesi L. Detection of on-target and off-target mutations generated by CRISPR/Cas9 and other sequence-specific nucleases. Biotechnol Adv. 2017;35:95-104.

61. Bae S, Kweon J, Kim HS, Kim J-S. Microhomology-based choice of Cas9 nuclease target sites. Nat Methods. 2014;11:705-6.

62. Vu GTH, Cao HX, Fauser F, Reiss B, Puchta H, Schubert I. Endogenous sequence patterns predispose the repair modes of CRISPR/Cas9-induced DNA double strand breaks in Arabidopsis thaliana. Plant J. 2017:92:57-67.

63. Yang Z, Steentoft C, Hauge C, Hansen L, Thomsen AL, Niola F, et al. Fast and sensitive detection of indels induced by precise gene targeting. Nucleic Acids Res. 2015:43:e59.

64. Mock U, Hauber I, Fehse B. Digital PCR to assess gene-editing frequencies (GEF-dPCR) mediated by designer nucleases. Nat Protoc. 2016;11:598-615.

65. Sander JD, Joung JK. CRISPR-Cas systems for editing, regulating and targeting genomes. Nat Biotechnol. 2014;32:347-55.

66. sgRNA Designer. https://portals.broadinstitute.org/gpp/public/analysis-tools/ sgrna-design. Accessed 16 Feb 2017.
67. WU-CRISPR. http://crispr.wustl.edu/. Accessed 16 Feb 2017.

68. Christensen AH, Sharrock RA, Quail PH. Maize polyubiquitin genes: structure, thermal perturbation of expression and transcript splicing, and promoter activity following transfer to protoplasts by electroporation. Plant Mol Biol. 1992;18:675-89.

69. Christensen AH, Quail PH. Ubiquitin promoter-based vectors for high-level expression of selectable and/or screenable marker genes in monocotyledonous plants. Transgenic Res. 1996;5:213-8.

70. Sasanuma T. Characterization of the rbcS multigene family in wheat: subfamily classification, determination of chromosomal location and evolutionary analysis. Mol Gen Genomics. 2001;265:161-71.

71. Yoo S-D, Cho Y-H, Sheen J. Arabidopsis mesophyll protoplasts: a versatile cell system for transient gene expression analysis. Nat Protoc. 2007;2:1565-72.

72. Langmead B, Salzberg S. Fast gapped-read alignment with bowtie 2. Nat Methods. 2012:9:357-9.

73. Li H, Handsaker B, Wysoker A, Fennell T, Ruan J, Homer N, et al. The sequence alignment/map format and SAMtools. Bioinformatics. 2009;25: 2078-9.

\section{Publisher's Note}

Springer Nature remains neutral with regard to jurisdictional claims in published maps and institutional affiliations.
Ready to submit your research? Choose BMC and benefit from:

- fast, convenient online submission

- thorough peer review by experienced researchers in your field

- rapid publication on acceptance

- support for research data, including large and complex data types

- gold Open Access which fosters wider collaboration and increased citations

- maximum visibility for your research: over $100 \mathrm{M}$ website views per year

At BMC, research is always in progress.

Learn more biomedcentral.com/submissions 